Article

\title{
Planetary Nebulae Embryo after a Common Envelope Event
}

\author{
Natalia Ivanova ${ }^{1, *(D)}$ and Jose L. A. Nandez ${ }^{1,2}$ (D) \\ 1 Department of Physics, University of Alberta, Edmonton, AB T6G 2E7, Canada; jnandez@sharcnet.ca \\ 2 SHARCNET, Faculty of Science, University of Western Ontario, London, ON N6A 5B7, Canada \\ * Correspondence: nata.ivanova@ualberta.ca
}

Received: 7 June 2018; Accepted: 9 July 2018; Published: 19 July 2018

\begin{abstract}
In the centers of some planetary nebulae are found close binary stars. The formation of those planetary nebulae was likely through a common envelope event, which transformed an initially-wide progenitor binary into the currently observed close binary, while stripping the outer layers away. A common envelope event proceeds through several qualitatively different stages, each of which ejects matter at its own characteristic speed, and with a different degree of symmetry. Here, we present how typical post-common envelope ejecta looks kinematically a few years after the start of a common envelope event. We also show some asymmetric features we have detected in our simulations (jet-like structures, lobes, and hemispheres).
\end{abstract}

Keywords: stellar evolution; binarity; planetary nebulae

\section{Introduction}

About 20 percent of planetary nebulae (PN) were likely formed as a result of a common envelope (CE) event that took place in the progenitor binary [1]. A CE event is an interaction during which one of the stars of the binary (the donor) shares its outer layers with its companion star (see for a review [2]). One of the possible outcomes of a CE event is the formation of a binary that is more compact than the initial binary, where one of the stars is the hot core of the initial donor star. In the case of binary formation, the shared CE is ejected; if the stripped core remains hot, the expelled CE may appear in the future as a PN.

About 50 post-CE PN with central stars are known [1,3,4]; CE events could be responsible for most non-spherical PNe [5]. Even though many post-CE PN are bipolar [4], the morphology of the post-CE PN is not uniform. The observed morphology may depend on the observation angle-e.g., an equatorial view favors a narrow-waist shape while a pole-on view favors a ring-type shape. Observed post-CE nebulae sometimes have jets and are elliptical in a shape (see the collection of various observed shapes of PNe in [1]). Not all of the observed non-spherical PNe have to be explained by a $\mathrm{CE}$ interaction, some could be a result of a triple interaction or be shaped by planets $[6,7]$. On the other hand, it has also been shown that a bipolar PN, which could be the most common shape for non-spherical PNe (see, e.g., [4]), could not have been formed during a single star's Asymptotic Giant Branch (AGB) evolution [8,9], where a binary is needed to provide the angular momentum to shape the final PN. This all hints that understanding of strong binary interactions during the envelope removal is important for an overall understanding of PN morphology.

Proper simulation of PN formation due to a CE event must include a self-consistent three-dimensional simulation of the $\mathrm{CE}$ event with complete ejection, and the follow-up evolution during which the ejecta becomes a PN. The latter is expected to include a long-term interaction with a pre-CE interstellar medium (presumably shaped by the previous episodes of mass loss by stellar winds), radiative cooling of the expanding envelope, stages of a fast wind, and re-ionization by a hot 
central star. Recent simulations by Garcia-Segura et al. [10] have shown that, indeed, a bipolar shape can be achieved as a result of considering the interaction of the envelope and a binary. The outer lobes are usually bipolar and the inner lobes are elliptical, bipolar or barrel-type. That study gave detailed attention to the late stages of post-CE evolution. For the initial conditions, they used CE simulations presented in [11]. These CE simulations were performed using the adaptive mesh refinement three-dimensional code FLASH 2.4 [12]. We note that the CE simulations used there as the initial conditions did not end with a complete CE ejection, and there is still some substantial envelope mass left behind, with an unknown fate.

In this contribution, we do the opposite: we provide the kinematic picture of very young, but complete, post-CE ejecta obtained using the smoothed particle hydrodynamics three-dimensional code StarSmasher [13,14]. A typical setup and description of successful CE events can be found in [15,16], but the evolution of the ejecta is limited to about three years. We stress that the expansion age of the ejecta that we can model is very small. It is much smaller not just compared to the age of a typical PNe, $\geq 10,000$ years, but also compared to the expansion age of the so-called protoplanetary, or preplanetary, nebulae [17], which is of the order of 3000 years [18]. We term therefore the post-CE ejecta a few years after a CE event as a PN embryo.

\section{The Stages of a CE Event, and the Associated Mass Outflows}

The outcome of a CE event is usually found using the energy formalism, first introduced by Webbink [19], Livio and Soker [20]. The original energy formalism balances the released orbital energy as an energy source, $E_{\text {orb,ini }}-E_{\text {orb,fin, }}$ with the required energy to dispel the bound envelope, $E_{\text {bind,env }}$, as the energy sink. It can be noted that, in this balance, the post-CE ejecta should have null velocity at infinity. Recently, the energy formalism was updated to take into account what fraction of the released orbital energy is taken away by the ejected envelope at infinity, $\alpha_{\mathrm{unv}}^{\infty}$, as well as the potentially available recombination energy, $E_{\text {rec }}[16]$ :

$$
\left(E_{\text {orb,ini }}-E_{\text {orb,fin }}\right)\left(1-\alpha_{\text {unb }}^{\infty}\right)+E_{\text {bind,env }}+E_{\text {rec }}=0
$$

Recombination energy is a "potential" energy in the sense that it is not readily available to drive an envelope expansion at the start of the CE event, but, after the recombination is triggered, it can be useful at the late stages of a CE event. Recombination energy can remove all the remaining bound parts of the envelope if the envelope material starts to recombine after it expands beyond the "recombination" radius (for the definition, see [21]). The discussion on whether the recombination energy can be used for driving the expansion of a CE, or it would be lost as radiation remains active. Recently, it was shown that neither of the two energy transfer mechanisms in stars-normal sub-sonic stellar convection or radiative diffusion-are capable of transferring the released recombination energy to the surface in most of the cases of regular (as opposed to self-regulated) CE events [22]. A regular $\mathrm{CE}$ event takes place on a dynamical timescale; during a regular $\mathrm{CE}$ event radiative losses from the surface are negligible compared to the energy released from shrinking orbit (or other energy sources inclusive of recombination). During a self-regulated stage of a CE event, first identified by Meyer and Meyer-Hofmeister [23], the energy loss via surface luminosity matches the energy source. During a self-regulated stage, the ionization profile by mass remains either constant, or propagates too slowly for recombination to affect the $\mathrm{CE}$ event energetics and outcomes.

In the published simulations of completed CE events, the ejecta was found to take away about $20-50 \%$ of the released orbital energy [16]. The corresponding mass-averaged velocity of the ejecta at infinity is in the range of $40-80 \mathrm{~km} / \mathrm{s}$. This is in principle higher than the observed expansion velocities, which are typically $<40 \mathrm{~km} / \mathrm{s}$ [24-26], but this does not yet account for future deceleration due to interactions with previously lost matter in the stellar wind, or with the interstellar medium.

The velocity of the ejected material is not constant across the ejecta-see the typical direction-averaged profile in Figure 1. Ivanova and Nandez [21] identified four kinds of matter ejection processes during a CE event. First is an "initial ejection", before the plunge (for exact definitions 
of various stages, see [21]). In the initial ejecta, most of the angular momentum is taken away, and the ejected material has a high velocity. The second ejecta are formed during the plunge, when the energy the $\mathrm{CE}$ material receives is mainly the mechanical energy transferred from the shrinking binary orbit. Once the envelope is puffed up and is no longer tidally coupled with the shrunken binary orbit, the plunge is slowed down. The envelope ejection is now driven primarily by the recombination energy and is termed as a recombination outflow. This fraction of the ejecta has almost uniform velocity; the rate of the mass loss in the analyzed simulations is $0.2-2 M_{\odot}$ year ${ }^{-1}$, but can be expected to vary in general. Finally, as the slowly expanding but still bound envelope evolves on its new parabolic trajectory, the bottom part of the envelope may fall back and trigger an additional dynamical ejection, termed as a "shell-triggered" ejection (not shown in Figure 1).

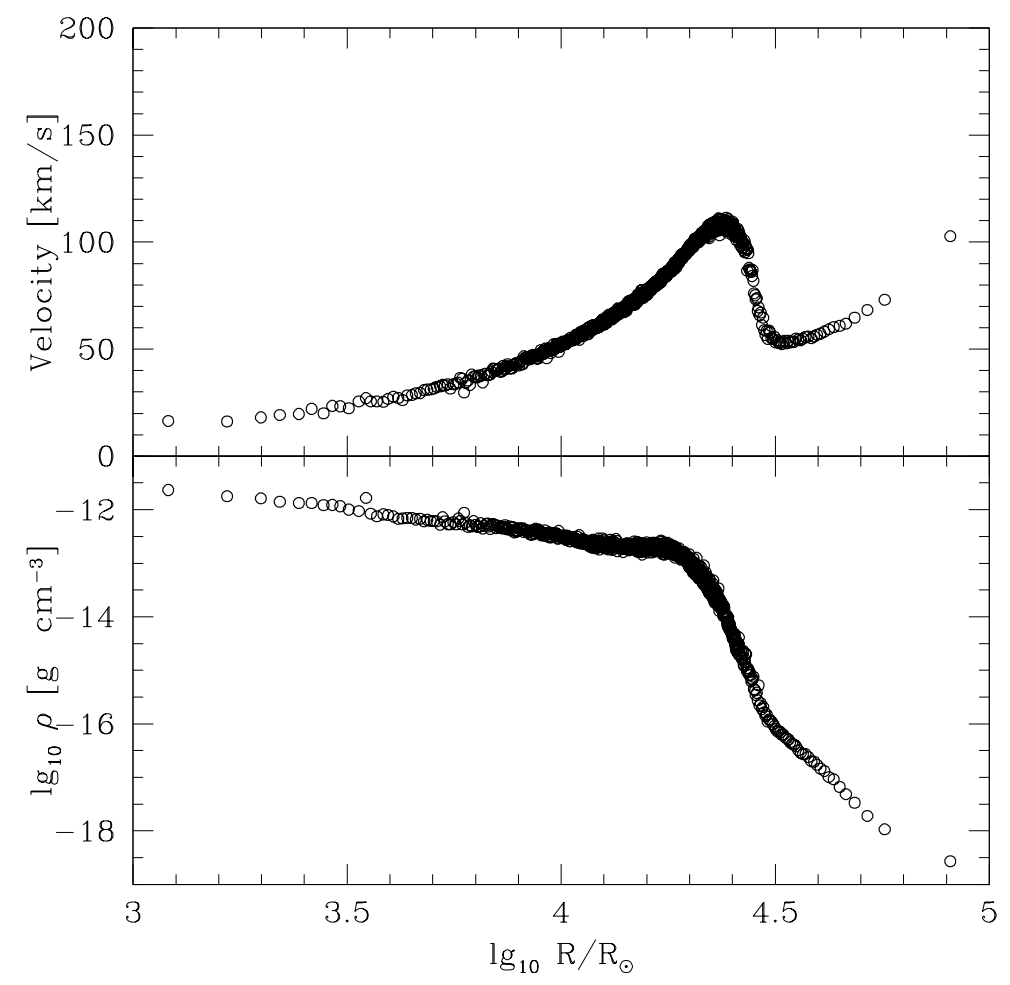

Figure 1. A typical profile of CE ejecta, weighted-by-mass averaged over all directions, shown using the CE simulation of an initial binary consisting of a $1.2 M_{\odot}$ red giant with a $0.32 M_{\odot}$ core and a $0.32 M_{\odot}$ white dwarf companion. The top panel shows velocity and the bottom panel shows density. The profile is shown 800 days after the plunge. There is no envelope material left within the inner $1000 R_{\odot}$.

If the rate of the envelope removal by recombination outflows is slow or ineffective, the system may evolve into a self-regulated spiral-in. During the self-regulated spiral-in, the envelope continues to lose material, by the combined work of recombination and pulsations [27,28]; a CE can also experience shell-triggered ejections similar to the case of a dynamical CE event. The mass loss is mainly driven during pulsations. This can only be modeled now using one-dimensional codes, and hence the kinematic profile of the ejecta during a self-regulated spiral-in is not entirely clear.

\section{Morphology of the CE Ejecta}

The variety-in terms of donor masses, radii at the start of a CE event, the companion masses, how well the stars' rotation are synchronized with the orbit, etc.- of the three-dimensional simulations performed to date with successful CE ejection is very limited, due to the heavy computational demand of those simulations. Besides, the existing three-dimensional simulations were performed for the case 
of red giant donors, while the most likely donors in the case of the observed PNe are AGB stars. In this respect, the results presented below are limited, and should not be expected to explain all plausible PNe morphology, but they may give us some clues. Here, we use the simulations that were described in [16]; the data presented here have not yet been published elsewhere. Simulations with a very low mass companion were performed for this contribution, using the same numerical methods as described in [16], using 200,000 particles for the donor star.

\subsection{Typical Shape-Spherical Symmetry}

The most typical shape of a post-CE ejecta, as found in three-dimensional simulations with red giant donors, is spherically symmetric. As a slice in density, it can be said to have a ring shape (see Figure 2), although one has to keep in mind that it is a two-dimensional representation of otherwise rather spherically symmetric object. The ring contains most of the mass, although the entire ejecta propagates well beyond the shown domain. This shape visually resembles the images of such post-CE nebulae as NGC 6337 and Sp 1, which were found to be post-CE PNe [29,30], or Hf 38, also argued to have two central stars (see the image in [5]). This visual symmetry of the ejecta is mainly due to the symmetry of recombination outflows. It is, however, difficult to fully separate how much symmetry was produced by the recombination outflows, and how much was already imprinted in the plunge-driven ejecta (for more discussion on the symmetry of angular momentum inside the $\mathrm{CE}$ during the plunge, see [21]). We note that in simulations that did not end with the complete removal of the envelope, e.g. in the simulations of the merger event V1309 Sco [31], the outflow was found to be less spherically symmetric, and resembles an actual ring, which would be seen as a circular ring only from a pole.

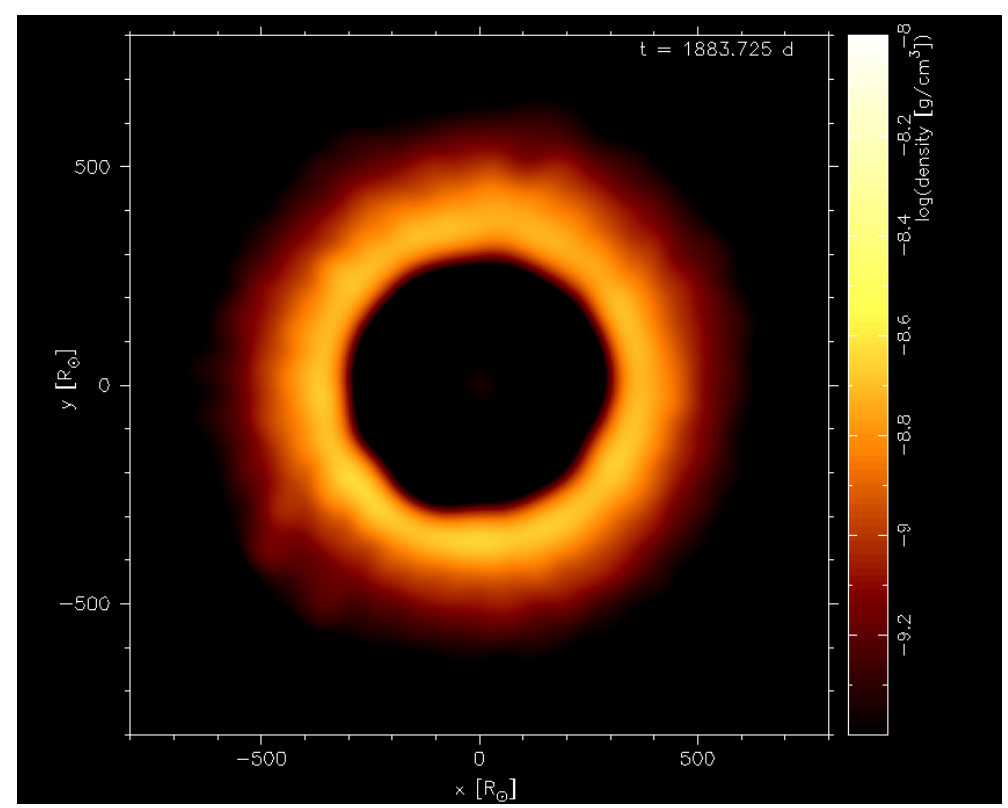

Figure 2. A typical post-CE nebula from a three-dimensional simulation. Here, we show the case of a $1.6 M_{\odot}$ red giant with a $0.32 M_{\odot}$ core and a $0.36 M_{\odot}$ white dwarf companion. Only material with densities above $10^{-9.3}$ is shown (the shown intensity scale starts at $10^{-9.4} \mathrm{~g} \mathrm{~cm}^{-3}$ and ends at $10^{-8} \mathrm{~g} \mathrm{~cm}^{-3}$ ). In the entire domain of simulation, there is no material with a higher density than used for the intensity scale, a lower density material surrounds the shown object (see also Figure 3 ). This is about 1000 days after the plunge. The shown domain is $1600 R_{\odot}$ by $1600 R_{\odot}$. This figure was created using ParaView [32]. 


\subsection{Deviations from Spherical Symmetry}

We find that deviations from a spherically symmetric shape are produced in the earlier stages of a $\mathrm{CE}$ event, either during the initial stage when most of the angular momentum is lost, or during the plunge. Noticeable deviations can include a jet-like formation in a polar direction (see Figures 3 and 4), accompanied by an underdense lobe cleared by the jet, and one or more hemispheric bubbles (see Figures 4 and 5).
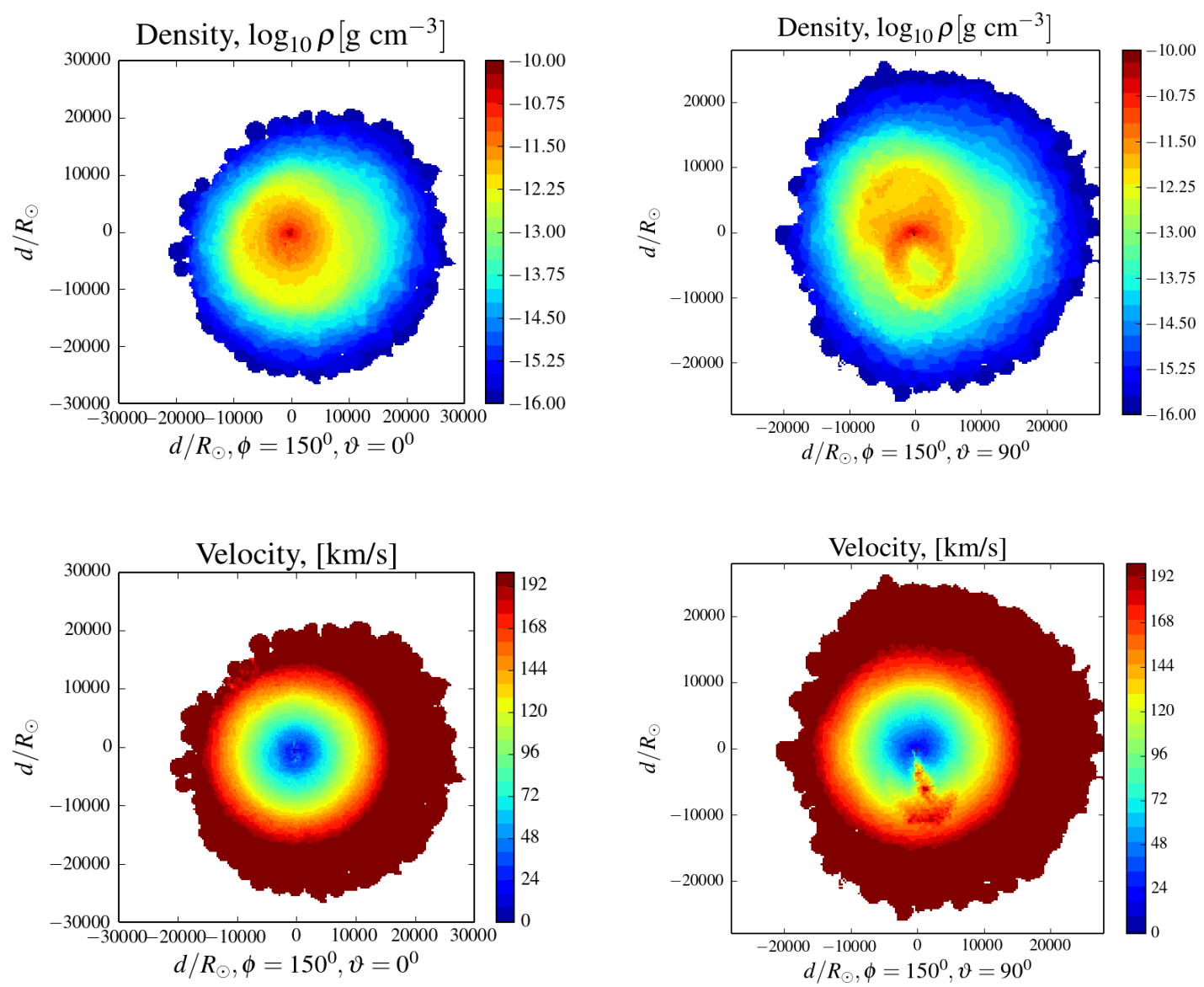

Figure 3. Density and velocity, in the equatorial $x y\left(\theta=0^{\circ}\right)$ and vertical $x^{\prime} z$ planes $\left(\theta=90^{\circ}\right)$. For these "slices", the plane goes through the origin, and then is first rotated by an angle $\phi$ around the axis $z$ to produce new axis $x^{\prime}$, and then by an angle $\theta$ around the axis $x^{\prime}$. The thickness of the plane is 0.1 of the size. $\phi$ rotation is chosen to bring the maximum symmetry in $x^{\prime} y$ plane for $\theta=0$. The matter with density below $10^{-16} \mathrm{~g} \mathrm{~cm}^{-3}$ is not shown. This CE simulation uses an initial binary consisting of a $1.2 M_{\odot}$ red giant with a $0.32 M_{\odot}$ core and a $0.32 M_{\odot}$ white dwarf companion. These slices are shown about 1000 days after the plunge. 

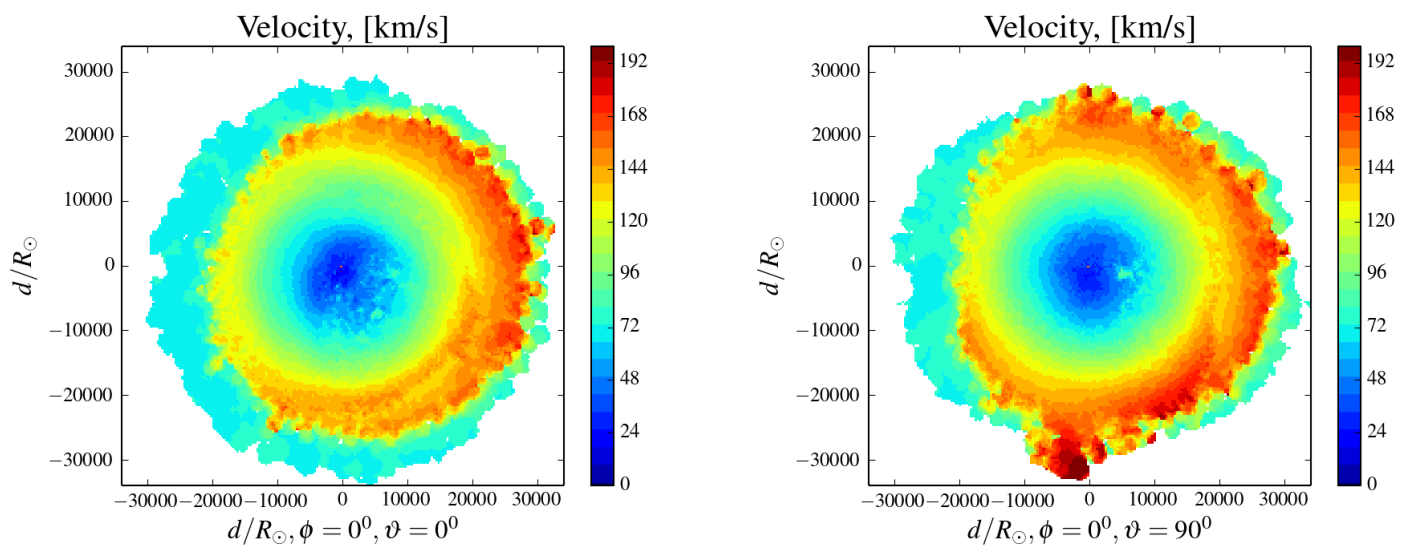

Figure 4. Velocity, in the equatorial $x y\left(\theta=0^{\circ}\right)$ and vertical $x^{\prime} z$ planes $\left(\theta=90^{\circ}\right)$, constructed similarly to Figure 3. This CE simulation uses an initial binary consisting of a $1.2 M_{\odot}$ red giant with a $0.32 M_{\odot}$ core and a $0.4 M_{\odot}$ white dwarf companion. This is about 800 days after the plunge.
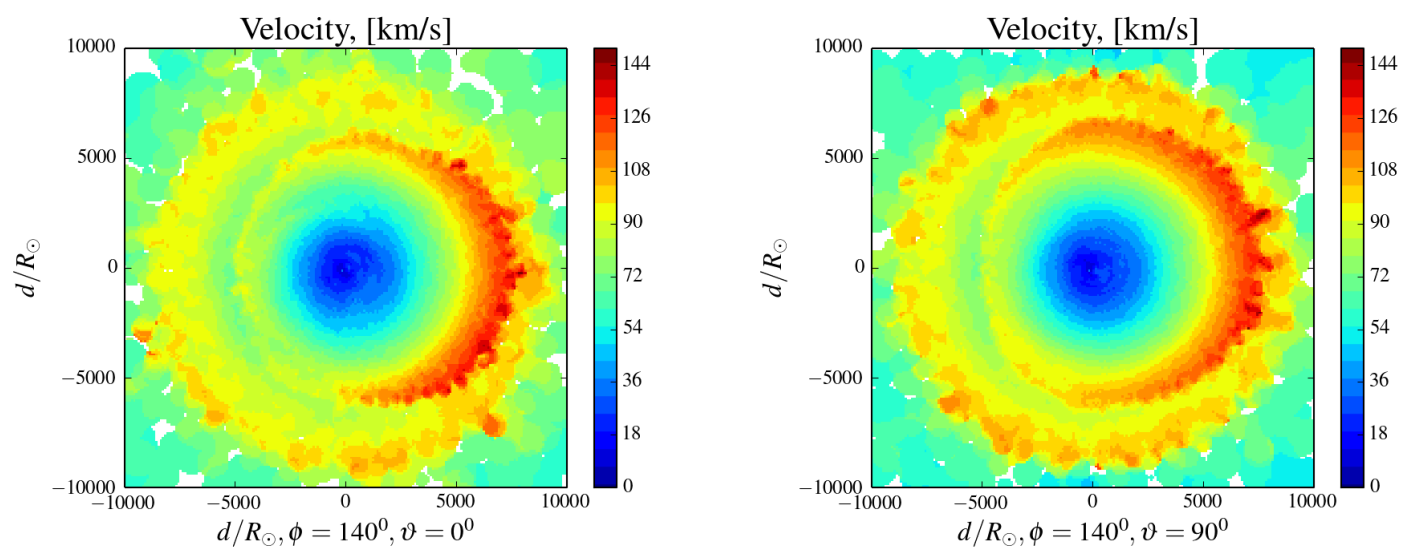

Figure 5. Velocity, in the equatorial $x y\left(\theta=0^{\circ}\right)$ and vertical $x^{\prime} z$ planes $\left(\theta=90^{\circ}\right)$, constructed similarly to Figure 3. This CE simulation uses an initial binary consisting of a $1.25 M_{\odot}$ red giant with a $0.41 M_{\odot}$ core and a $0.067 M_{\odot}$ white dwarf companion. This is about 1000 days after the plunge.

\section{Discussion}

Analyzing the simulations of successful CE events with red giant donors, we find that post-CE ejecta are not uniform: neither in the average expansion velocity across the ejecta, nor in their density. Earlier, faster and lower-density ejecta is less spherically symmetric and can feature jet-like formations, spherical shells, hemispherical bubbles, and lobes. Later, slower but more dense ejecta is more spherically symmetric. Its symmetrical features are likely due to the inherent symmetry of the recombination outflows that drive it, or they can be produced at a late stage of the plunge. An asymmetry in the late ejecta could be produced by shell-triggered ejections.

The considered ejecta, regarding their expansion ages, are just embryos of possible future PNe. During the considered early stages of the post-CE expansion, we do not detect the bipolar shape typical of many observed PNe. The bipolar shape could be produced if additional physics is taken into account during subsequent stages of ejecta evolution (see, e.g., [10]). In addition, the cases modeled so far are not directly related to CE events in binaries with AGB donors, where the envelopes are less bound than the donors we have considered, and hence these results should be taken with a grain of salt. 
An essential point is that kinematic properties of the post-CE ejecta are imprinted by several stages of the CE event that formed it, as well as by the total energy that the ejecta takes away. We hope, therefore, that kinematic studies of the observed PNe and preplanetary nebulae could help us to substantially constrain the physics of CE events.

Author Contributions: N.I. and J.N. conceived and designed the simulations; J.N. performed the simulations; and N.I. analyzed the data and wrote the paper.

Funding: N.I. acknowledges support from CRC program, and funding from an NSERC Discovery Grant.

Acknowledgments: This research was enabled by the use of computing resources provided by WestGrid, Sharcnet and Compute/Calcul Canada.

Conflicts of Interest: The authors declare no conflict of interest.

\section{Abbreviations}

The following abbreviations are used in this manuscript:

AGB Asymptotic Giant Branch

PN planetary nebula

CE common envelope

\section{References}

1. De Marco, O.; Reichardt, T.; Iaconi, R.; Hillwig, T.; Jacoby, G.H.; Keller, D.; Izzard, R.G.; Nordhaus, J.; Blackman, E.G. Post-common envelope PN, fundamental or irrelevant? In Planetary Nebulae: Multi-Wavelength Probes of Stellar and Galactic Evolution; Liu, X., Stanghellini, L., Karakas, A., Eds.; International Astronomical Union: Paris, France, 2017; Volume 323, pp. 213-217. [CrossRef]

2. Ivanova, N.; Justham, S.; Chen, X.; De Marco, O.; Fryer, C.L.; Gaburov, E.; Ge, H.; Glebbeek, E.; Han, Z.; Li, X.D.; et al. Common envelope evolution: Where we stand and how we can move forward. Astron. Astrophys. Rev. 2013, 21, 59. [CrossRef]

3. Miszalski, B.; Acker, A.; Moffat, A.F.J.; Parker, Q.A.; Udalski, A. Binary planetary nebulae nuclei towards the Galactic bulge. I. Sample discovery, period distribution, and binary fraction. Astron. Astrophys. 2009, 496, 813-825. [CrossRef]

4. Miszalski, B.; Acker, A.; Parker, Q.A.; Moffat, A.F.J. Binary planetary nebulae nuclei towards the Galactic bulge. II. A penchant for bipolarity and low-ionisation structures. Astron. Astrophys. 2009, 505, 249-263. [CrossRef]

5. Barker, H.; Zijlstra, A.; De Marco, O.; Frew, D.J.; Drew, J.E.; Corradi, R.L.M.; Eislöffel, J.; Parker, Q.A. The binary fraction of planetary nebula central stars-III. the promise of VPHAS ${ }^{+}$. Mon. Not. R. Astron. Soc. 2018, 475, 4504-4523. [CrossRef]

6. Bear, E.; Soker, N. Planetary Nebulae that Cannot Be Explained by Binary Systems. Astrophys. J. Lett. 2017, 837, L10. [CrossRef]

7. Sabach, E.; Soker, N. Accounting for planet-shaped planetary nebulae. Mon. Not. R. Astron. Soc. 2018, 473, 286-294. [CrossRef]

8. García-Segura, G.; Villaver, E.; Langer, N.; Yoon, S.C.; Manchado, A. Single Rotating Stars and the Formation of Bipolar Planetary Nebula. Astrophys. J. 2014, 783, 74. [CrossRef]

9. García-Segura, G.; Villaver, E.; Manchado, A.; Langer, N.; Yoon, S.C. Rotating Stars and the Formation of Bipolar Planetary Nebulae. II. Tidal Spin-up. Astrophys. J. 2016, 823, 142. [CrossRef]

10. Garcia-Segura, G.; Ricker, P.M.; Taam, R.E. Common Envelope Shaping of Planetary Nebulae. ArXiv 2018, arXiv:astro-ph.SR/1804.09309.

11. Ricker, P.M.; Taam, R.E. An AMR Study of the Common-envelope Phase of Binary Evolution. Astrophys. J. 2012, 746, 74. [CrossRef]

12. Fryxell, B.; Olson, K.; Ricker, P.; Timmes, F.X.; Zingale, M.; Lamb, D.Q.; MacNeice, P.; Rosner, R.; Truran, J.W.; Tufo, H. FLASH: An Adaptive Mesh Hydrodynamics Code for Modeling Astrophysical Thermonuclear Flashes. Astrophys. J. Suppl. Ser. 2000, 131, 273-334. [CrossRef] 
13. Gaburov, E.; Lombardi, J.C., Jr.; Portegies Zwart, S. On the onset of runaway stellar collisions in dense star clusters-II. Hydrodynamics of three-body interactions. Mon. Not. R. Astron. Soc. Lett. 2010, 402, 105-126. [CrossRef]

14. Gaburov, E.; Lombardi, J.C., Jr.; Portegies Zwart, S.; Rasio, F.A. StarSmasher: Smoothed Particle Hydrodynamics Code for Smashing Stars and Planets; Astrophysics Source Code Library: Houghton, MI, USA, 2018.

15. Nandez, J.L.A.; Ivanova, N.; Lombardi, J.C. Recombination energy in double white dwarf formation. Mon. Not. R. Astron. Soc. Lett. 2015, 450, L39-L43. [CrossRef]

16. Nandez, J.L.A.; Ivanova, N. Common envelope events with low-mass giants: understanding the energy budget. Mon. Not. R. Astron. Soc. 2016, 460, 3992-4002. [CrossRef]

17. Volk, K.M.; Kwok, S. Evolution of protoplanetary nebulae. Astrophys. J. 1989, 342, 345-363. [CrossRef]

18. Sahai, R.; Sánchez Contreras, C.; Morris, M. A Starfish Preplanetary Nebula: IRAS 19024+0044. Astrophys. J. 2005, 620, 948-960. [CrossRef]

19. Webbink, R.F. Double white dwarfs as progenitors of R Coronae Borealis stars and Type I supernovae. Astrophys. J. 1984, 277, 355-360. [CrossRef]

20. Livio, M.; Soker, N. The common envelope phase in the evolution of binary stars. Astrophys. J. 1988, 329, 764-779. [CrossRef]

21. Ivanova, N.; Nandez, J.L.A. Common envelope events with low-mass giants: understanding the transition to the slow spiral-in. Mon. Not. R. Astron. Soc. 2016, 462, 362-381. [CrossRef]

22. Ivanova, N. On the Use of Hydrogen Recombination Energy during Common Envelope Events. Astrophys. J. Lett. 2018, 858, L24. [CrossRef]

23. Meyer, F.; Meyer-Hofmeister, E. Formation of cataclysmic binaries through common envelope evolution. Astron. Astrophys. 1979, 78, 167-176.

24. Weinberger, R. A catalogue of expansion velocities of Galactic planetary nebulae. Astron. Astrophys. Suppl. Ser. 1989, 78, 301-324.

25. Gesicki, K.; Zijlstra, A.A. Expansion velocities and dynamical ages of planetary nebulae. Astron. Astrophys. 2000, 358, 1058-1068.

26. Arnaboldi, M.; Doherty, M.; Gerhard, O.; Ciardullo, R.; Aguerri, J.A.L.; Feldmeier, J.J.; Freeman, K.C.; Jacoby, G.H. Expansion Velocities and Core Masses of Bright Planetary Nebulae in the Virgo Cluster. Astrophys. J. Lett. 2008, 674, L17. [CrossRef]

27. Ivanova, N.; Justham, S.; Podsiadlowski, P. On the role of recombination in common-envelope ejections. Mon. Not. R. Astron. Soc. 2015, 447, 2181-2197. [CrossRef]

28. Clayton, M.; Podsiadlowski, P.; Ivanova, N.; Justham, S. Episodic mass ejections from common-envelope objects. Mon. Not. R. Astron. Soc. 2017, 470, 1788-1808. [CrossRef]

29. García-Díaz, M.T.; Clark, D.M.; López, J.A.; Steffen, W.; Richer, M.G. The Outflows and Three-Dimensional Structure of NGC 6337: A Planetary Nebula with a Close Binary Nucleus. Astrophys. J. 2009, 699, 1633-1638. [CrossRef]

30. Hillwig, T.C.; Jones, D.; De Marco, O.; Bond, H.E.; Margheim, S.; Frew, D. Observational Confirmation of a Link Between Common Envelope Binary Interaction and Planetary Nebula Shaping. Astrophys. J. 2016, 832, 125. [CrossRef]

31. Nandez, J.L.A.; Ivanova, N.; Lombardi, J.C., Jr. V1309 Sco-Understanding a Merger. Astrophys. J. 2014, 786, 39. [CrossRef]

32. Ayachit, U. The ParaView Guide: A Parallel Visualization Application; Kitware, Inc.: New York, NY, USA, 2015.

(c) 2018 by the authors. Licensee MDPI, Basel, Switzerland. This article is an open access article distributed under the terms and conditions of the Creative Commons Attribution (CC BY) license (http:/ / creativecommons.org/licenses/by/4.0/). 